KA-THEP-1-1995

UAB-FT-357

January 1995

\title{
STRONG SUPERSYMMETRIC QUANTUM EFFECTS ON THE TOP QUARK WIDTH
}

\author{
Andreas DABELSTEIN, Wolfgang HOLLIK, Christoph JÜNGER \\ Institut für Theoretische Physik, \\ Universität Karlsruhe, D-76128 Karlsruhe, Germany \\ Ricardo A. JIMÉNEZ, Joan SOLÀ \\ Grup de Física Teòrica \\ and \\ Institut de Física d'Altes Energies \\ Universitat Autònoma de Barcelona \\ 08193 Bellaterra (Barcelona), Catalonia, Spain
}

\begin{abstract}
We compute the one-loop supersymmetric QCD quantum effects on the width $\Gamma\left(t \rightarrow W^{+} b\right)$ of the canonical main decay of the top quark within the framework of the MSSM. The corrections can be of either sign depending on whether the stop squark mass is above or below the top quark decay threshold into stop and gluino $\Gamma(t \rightarrow \tilde{t} \tilde{g})$. For $m_{\tilde{t}}$ above that threshold, the corrections are negative and can be of the same order (and go in the same direction) as the ordinary QCD corrections, even for stop and gluino masses of $\mathcal{O}(100) \mathrm{GeV}$. Since the electroweak supersymmetric quantum effects turn out to be also of the same sign and could be of the same order of magnitude, the total MSSM correction to the top quark width could potentially result in a rather large $\mathcal{O}(10-25) \%$ reduction of $\Gamma\left(t \rightarrow W^{+} b\right)$ far beyond the conventional QCD expectations.
\end{abstract}




\section{Introduction}

In spite of being a sequential fermion the top quark plays a special role in the families of fermions. The Standard Model predicts a fairly strong direct interaction with the Higgs sector through a large Yukawa coupling distinguishing the top quark as a particularly helpful probe for the electroweak symmetry breaking mechanism. It may also be a useful laboratory to unravel effects beyond the Standard Model such as those from the minimal supersymmetric standard model (MSSM) [1]. For realizing possible hints for new physics of this class of extensions in future precise measurements of the top quark properties detailed predictions are necessary including higher order terms. The quantum effects to the conventional top width $\Gamma\left(t \rightarrow W^{+} b\right)$ induced by the one-loop radiative corrections from the chargino, neutralino and scalar fermion sector of the MSSM have been studied in detail in [2] (see also [3]) with the main result that there can be relatively large corrections from this "genuine" electroweak SUSY part of the MSSM (up to 10\%) depending sensitively on the range of the model parameters. These potentially large contributions are of the same (negative) sign as the standard QCD corrections [4, 5] and of the same order of magnitude. This is different from the standard electroweak corrections which have been shown to be small with very little variation on the mass of the top quark and the Higgs boson [5, 6, 7]. Also the non-standard loop effects from the SUSY Higgs sector are well below $1 \%$, 8 , 9].

In this paper we complete the discussion of the one-loop quantum effects on the top quark width in the framework of the MSSM by computing the missing strong SUSY corrections induced by virtual gluinos and scalar quarks. Due to the strength of the strong coupling constant these additional loop effects are expected to be comparable with the electroweak terms arising from large Yukawa couplings if the gluino and squark masses are not too high. Our study shows that the most interesting virtual effects (i.e. when the direct real decay channel $t \rightarrow \tilde{t} \tilde{g}$ is kinematically forbidden) have a similar signature: the SUSY QCD contributions are negative and can reach several percent, thus enhancing the conventional QCD corrections nearly by a factor of two.

\section{Supersymmetric QCD corrections}

To compute the one-loop QCD corrections to $\Gamma_{t} \equiv \Gamma\left(t \rightarrow W^{+} b\right)$ in the MSSM, we shall adopt the on-shell renormalization scheme, where the fine structure constant, $\alpha$, and the masses of the gauge bosons, fermions and scalars are the renormalized parameters: 
$\left(\alpha, M_{W}, M_{Z}, M_{H}, m_{f}, M_{S U S Y}, \ldots\right)$ 円. There are no universal supersymmetric corrections from the strong interaction at 1-loop order. The flavor specific vertex corrections and quark self-energies originating from virtual gluinos and squarks (stop and sbottom species) are depicted in Fig.1. The SUSY-QCD interaction Lagrangian relevant to our calculation is given, in four-component notation, by

$$
\mathcal{L}=-\frac{g_{s}}{\sqrt{2}}\left[\tilde{q}_{L}^{i *}\left(\lambda_{r}\right)_{i j} \overline{\tilde{g}}^{r} P_{L} q^{j}-\bar{q}^{i}\left(\lambda_{r}\right)_{i j} P_{L} \tilde{g}^{r} \tilde{q}_{R}^{j}\right]+\text { h.c. }
$$

where $\tilde{g}^{r}(r=1,2, \ldots, 8)$ are the Majorana gluino fields, $\left(\lambda_{r}\right)_{i j}(i, j=1,2,3)$ are the GellMann matrices, and $\tilde{q}^{\prime}{ }_{a}=\left\{\tilde{q}_{L}, \tilde{q}_{R}\right\}$ are the weak-eigenstate squarks associated to the two chiral components $P_{L, R} q=1 / 2\left(1 \mp \gamma_{5}\right) q$; they are related to the corresponding masseigenstates $\tilde{q}_{a}$ by a rotation $2 \times 2$ matrix (we neglect intergenerational mixing):

$$
\begin{aligned}
\tilde{q}_{a} & =\sum_{b} R_{a b}^{(q)}{\tilde{q^{\prime}}}_{b}, \\
R^{(q)} & =\left(\begin{array}{cc}
\cos \theta_{q} & \sin \theta_{q} \\
-\sin \theta_{q} & \cos \theta_{q}
\end{array}\right) \quad(q=t, b)
\end{aligned}
$$

Using minimal field renormalization [10] (viz. one renormalization constant per symmetry multiplet), the renormalized vertex can be written in the notation of Ref. [2] as follows:

$$
\Gamma_{\mu}^{(1)}=i \frac{g}{\sqrt{2}}\left[\gamma_{\mu} P_{L}\left(1+F_{L}+\delta Z_{L}-\frac{1}{2} \hat{\Pi}_{t}\left(m_{t}^{2}\right)\right)+\gamma_{\mu} P_{R} F_{R}+\frac{p_{\mu}}{M_{W}}\left(P_{L} H_{L}+P_{R} H_{R}\right)\right]
$$

where the correction has been parametrized in terms of four form factors $F_{L}, F_{R}, H_{L}$ and $H_{R}$. The renormalized one-loop vertex is obtained from $\mathcal{L} \rightarrow \mathcal{L}+\delta \mathcal{L}$, where $\delta \mathcal{L}$ is calculated from $Z_{L}$ being the renormalization constant of the $(t, b)$ doublet:

$$
\left(\begin{array}{c}
t \\
b
\end{array}\right) \rightarrow Z_{L}^{1 / 2}\left(\begin{array}{c}
t \\
b
\end{array}\right)
$$

Fixing $Z_{L}=1+\delta Z_{L}$ through the condition of residue one for the renormalized bottom quark propagator

$$
\left.\frac{1}{\not k-m_{b}} \hat{\Sigma}^{b}(k) u_{b}(k)\right|_{\not k \rightarrow m_{b}}=0
$$

yields for $\delta Z_{L}$

$$
\delta Z_{L}=\Sigma_{L}^{b}\left(m_{b}^{2}\right)+m_{b}^{2}\left[\Sigma_{L}^{b^{\prime}}\left(m_{b}^{2}\right)+\Sigma_{R}^{b \prime}\left(m_{b}^{2}\right)+2 \Sigma_{S}^{b^{\prime}}\left(m_{b}^{2}\right)\right]
$$

and a finite wave-function renormalization for the external top quark line (in the framework of 10$]$ ):

$$
\hat{\Pi}_{t}\left(m_{t}^{2}\right)=\Pi_{t}\left(m_{t}^{2}\right)+\delta Z_{L},
$$

\footnotetext{
${ }^{1}$ For a comprehensive review, see e.g. refs. [10, 11.
} 
with $\Pi_{t}\left(m_{t}^{2}\right)$ given by

$$
\Pi_{t}\left(m_{t}^{2}\right)=-\Sigma_{L}^{t}\left(m_{t}^{2}\right)-m_{t}^{2}\left[\Sigma_{L}^{t \prime}\left(m_{t}^{2}\right)+\Sigma_{R}^{t}{ }^{\prime}\left(m_{t}^{2}\right)+2 \Sigma_{S}^{t^{\prime}}\left(m_{t}^{2}\right)\right] .
$$

$\delta Z_{L}$ and $\hat{\Pi}_{t}$ are obtained from the self energy diagrams of Fig.1, for which we used the decomposition

$$
\Sigma^{q}(p)=\Sigma_{L}^{q}\left(p^{2}\right) \not p P_{L}+\Sigma_{R}^{q}\left(p^{2}\right) \not p P_{R}+m_{q} \Sigma_{S}^{q}\left(p^{2}\right)
$$

and the notation $\Sigma^{\prime}(p) \equiv \partial \Sigma(p) / \partial p^{2}$. Explicitly, the gluino-squark contributions read

$$
\begin{aligned}
\Sigma_{L}^{q}\left(p^{2}\right) & =\frac{\alpha_{s}}{3 \pi}\left[\left(1+\cos 2 \theta_{q}\right) B_{1}\left(p^{2} ; m_{\tilde{g}}, m_{\tilde{q}_{1}}\right)+\left(1-\cos 2 \theta_{q}\right) B_{1}\left(p^{2} ; m_{\tilde{g}}, m_{\tilde{q}_{2}}\right)\right] \\
\Sigma_{R}^{q}\left(p^{2}\right) & =\frac{\alpha_{s}}{3 \pi}\left[\left(1-\cos 2 \theta_{q}\right) B_{1}\left(p^{2} ; m_{\tilde{g}}, m_{\tilde{q}_{1}}\right)+\left(1+\cos 2 \theta_{q}\right) B_{1}\left(p^{2} ; m_{\tilde{g}}, m_{\tilde{q}_{2}}\right)\right] \\
\Sigma_{S}^{q}\left(p^{2}\right) & =\frac{\alpha_{s}}{3 \pi}\left[\frac{m_{\tilde{g}}}{m_{q}} \sin 2 \theta_{q}\left(B_{0}\left(p^{2} ; m_{\tilde{g}}, m_{\tilde{q}_{1}}\right)-B_{0}\left(p^{2} ; m_{\tilde{g}}, m_{\tilde{q}_{2}}\right)\right)\right],
\end{aligned}
$$

$\theta_{q}$ being the left-right mixing angle defined in (2) which diagonalizes the $\tilde{q}$-squark mass matrix $\mathcal{M}_{\tilde{q}}^{2}(\tilde{q}=\tilde{t}, \tilde{b})$. As for the vertex diagram of Fig.1, the generic contribution to the form factors in eq.(3) is given by

$$
\begin{aligned}
F_{L}= & \frac{\alpha_{s}}{\pi} C_{F} a_{3} a_{1 L} a_{2 R} C_{00} \\
F_{R}= & \frac{\alpha_{s}}{\pi} C_{F} a_{3} a_{1 R} a_{2 L} C_{00} \\
H_{L}= & \frac{\alpha_{s}}{\pi} C_{F} a_{3} M_{W}\left[-a_{1 L} a_{2 L} m_{\tilde{g}}\left(C_{0}+C_{1}+C_{2}\right)\right. \\
& \left.+a_{1 R} a_{2 L} m_{t}\left(C_{1}+C_{11}+C_{12}\right)+a_{1 L} a_{2 R} m_{b}\left(C_{2}+C_{12}+C_{22}\right)\right] \\
H_{R}= & \frac{\alpha_{s}}{\pi} C_{F} a_{3} M_{W}\left[-a_{1 R} a_{2 R} m_{\tilde{g}}\left(C_{0}+C_{1}+C_{2}\right)\right. \\
& \left.+a_{1 L} a_{2 R} m_{t}\left(C_{1}+C_{11}+C_{12}\right)+a_{1 R} a_{2 L} m_{b}\left(C_{2}+C_{12}+C_{22}\right)\right] .
\end{aligned}
$$

where the colour factor $C_{F}$ is $4 / 3$. The notations and conventions for $B_{0}, B_{1}$ and the various three-point functions $C_{\ldots}=C_{\ldots}\left(p^{2},\left(p-p^{\prime}\right)^{2}, p^{2}, m_{\tilde{g}}, m_{1}, m_{2}\right)$ were adopted from [11]. There are four contributions of the type (11), which we shall denote with the indices $(k, l)=(1,1),(1,2),(2,1),(2,2)$. The corresponding masses and couplings associated to these contributions can be written in a compact form as follows:

$$
\begin{aligned}
m_{1} & =m_{\tilde{t}_{k}}, \quad m_{2}=m_{\tilde{b}_{l}}, \\
a_{3} & =(-1)^{k+l} \sin \left(\theta_{t}-k \frac{\pi}{2}\right) \sin \left(\theta_{b}-l \frac{\pi}{2}\right), \\
a_{1 L} & =(-1)^{k+1} \sin \left(\theta_{t}-k \frac{\pi}{2}\right), \\
a_{2 L} & =(-1)^{l+1} \cos \left(\theta_{b}-l \frac{\pi}{2}\right), \\
a_{1 R} & =(-1)^{k+1} \cos \left(\theta_{t}-k \frac{\pi}{2}\right), \\
a_{2 R} & =(-1)^{l+1} \sin \left(\theta_{b}-l \frac{\pi}{2}\right) .
\end{aligned}
$$


To obtain the result for $\Gamma\left(t \rightarrow b W^{+}\right)$we define, in analogy to Ref.[正], the four standard matrix elements

$$
\begin{aligned}
& M_{0}=\bar{u}_{b}\left(p^{\prime}\right) \notin P_{L} u_{t}(p) \\
& M_{1}=\bar{u}_{b}\left(p^{\prime}\right) \notin P_{R} u_{t}(p) \\
& M_{2}=\bar{u}_{b}\left(p^{\prime}\right) P_{L} u_{t}(p)(\epsilon \cdot p) / M_{W} \\
& M_{3}=\bar{u}_{b}\left(p^{\prime}\right) P_{R} u_{t}(p)(\epsilon \cdot p) / M_{W} .
\end{aligned}
$$

Then the one loop width can be written in terms of the Born width $\Gamma_{0}$ and

$$
\begin{aligned}
& G_{0}=\Sigma_{p o l}\left|M_{0}\right|^{2}=m_{t}^{2}+m_{b}^{2}-2 M_{W}^{2}+\frac{\left(m_{t}^{2}-m_{b}^{2}\right)^{2}}{M_{W}^{2}}, \\
& G_{1}=\Sigma_{p o l} M_{0} M_{1}^{*}=-6 m_{t} m_{b}, \\
& G_{2}=\Sigma_{p o l} M_{0} M_{2}^{*}=-\frac{m_{t}}{M_{W}}\left[m_{t}^{2}+m_{b}^{2}-\frac{M_{W}^{2}}{2}-\frac{\left(m_{t}^{2}-m_{b}^{2}\right)^{2}}{2 M_{W}^{2}}\right], \\
& G_{3}=\Sigma_{p o l} M_{0} M_{3}^{*}=\frac{m_{b}}{m_{t}} G_{2},
\end{aligned}
$$

and gives

$$
\begin{aligned}
\Gamma= & \Gamma_{0}\left(1+2 \operatorname{Re} F_{L}+2 \operatorname{Re} \delta Z_{L}-\operatorname{Re} \hat{\Pi}_{t}\left(m_{t}^{2}\right)\right) \\
& \left.+2 \frac{G_{1}}{G_{0}} \operatorname{Re} F_{R}+2 \frac{G_{2}}{G_{0}} \operatorname{Re} H_{L}+2 \frac{G_{3}}{G_{0}} \operatorname{Re} H_{R}\right),
\end{aligned}
$$

with 9

$$
\begin{aligned}
\Gamma_{0} & =\left(\frac{G_{F} M_{W}^{2}}{8 \pi \sqrt{2}}\right) m_{t}\left|V_{t b}\right|^{2} \frac{G_{0} \lambda^{1 / 2}\left(m_{t}^{2}, M_{W}^{2}, m_{b}^{2}\right)}{m_{t}^{4}} \\
& \simeq\left(\frac{G_{F} m_{t}^{3}}{8 \pi \sqrt{2}}\right)\left(1-\frac{M_{W}^{2}}{m_{t}^{2}}\right)^{2}\left(1+2 \frac{M_{W}^{2}}{m_{t}^{2}}\right) .
\end{aligned}
$$

A correction term from $\Delta r$ does not appear due to the absence of 1-loop SUSY QCD corrections in $\mu$-decay.

\section{Numerical analysis}

We are now in position to analyze the relative correction

$$
\delta_{\tilde{g}}=\frac{\Gamma-\Gamma_{0}}{\Gamma_{0}}
$$

induced by the strong virtual gluino effects. The corresponding supersymmetric electroweak corrections induced by charginos, neutralinos and sfermions have already been

\footnotetext{
${ }^{2} \lambda(x, y, z)=x^{2}+y^{2}+z^{2}-2(x y+x z+y z)$
} 
addressed in Refs. [2, 3], and will be briefly commented later on. The numerical analysis of the strong supersymmetric corrections is displayed in Figs.2-6. We used

$$
\alpha_{s}\left(m_{t}\right)=0.11
$$

as an input for the strong coupling constant, which remains essentially unchanged within the CDF range for the top quark mass: $160 \mathrm{GeV} \leq m_{t} \leq 190 \mathrm{GeV}$ [12]. Whenever a fixed value for $m_{t}$ is chosen, we take the central CDF value $m_{t}=174 \mathrm{GeV}$. In Figs.2a-2b we plot $\delta_{\tilde{g}}$ as a function of $m_{\tilde{g}}$ and of $m_{t}$, respectively. For the squark mass spectrum we have borrowed the standard pattern expected from models with radiatively induced breaking of the electroweak symmetry such as supergravity inspired models [1]:

$$
m_{\tilde{q}_{L, R}}^{2}=m_{q}^{2}+M_{\tilde{q}_{L, R}}^{2} \pm \cos 2 \beta\left(T_{L, R}^{3}-Q_{\tilde{q}} s_{w}^{2}\right) M_{Z}^{2}
$$

where $T_{L, R}^{3}$ and $Q_{\tilde{q}}$ stand, respectively, for the third component of weak isospin and electric charge corresponding to each member of the multiplet and for each "chiral" species $\tilde{q}_{L, R}$ of squarks. In that equation $\tan \beta$ stands for the ratio $v_{2} / v_{1}$ of vacuum expectation values giving masses to the $T^{3}=+1 / 2\left(v_{2}\right)$ and the $T^{3}=-1 / 2\left(v_{1}\right)$ components in each fermion doublet. Finally, the parameters $M_{\tilde{q}_{L, R}}$ are soft SUSY-breaking mass terms [1]. The mass splitting between the $T^{3}=+1 / 2$ and the $T^{3}=-1 / 2$ components is independent of $M_{\tilde{q}_{L}}$ because of $S U(2)_{L}$-gauge invariance, which requires $M_{\tilde{t}_{L}}=M_{\tilde{b}_{L}}$. For the $(t, b)$ doublet we have

$$
m_{\tilde{t}_{L}}^{2}-m_{\tilde{b}_{L}}^{2}=m_{t}^{2}+M_{W}^{2} \cos 2 \beta
$$

where the bottom quark mass is neglected. The previous equations refer to truly mass eigenvalues for the squarks only in the absence of mixing. Thus, in Fig.2 we assumed zero mixing for both stop and sbottom mass matrices. The effect of the mixing will be examined in a second step. Furthermore, since $M_{\tilde{q}_{R}}$ is independent of $M_{\tilde{q}_{L}}$, we may assume for simplicity that the $R$-type and $L$-type species are degenerate in mass. (This assumption will be dropped for stop squarks in the presence of mixing). In Fig.3, on the other hand, we test the sensitivity of the SUSY-QCD corrections on $\tan \beta$, which is very weak since this parameter enters only through the squark mass formula (19). Thus we shall take $\tan \beta=1$ for the other plots. This is in contrast to the electroweak SUSY corrections, which are very sensitive to $\tan \beta$, for it may induce large supersymmetric Yukawa couplings [2]. From Figs.2-3, it is clear that in the absence of mixing the strong SUSY corrections are typically of the order of $-1 \%$. For light gluinos of $\mathcal{O}(1) \mathrm{GeV} 13$, the correction is $2-3$ times higher (in absolute value) than for $\mathcal{O}(100) \mathrm{GeV}$ gluinos, but it never goes beyond $-2 \%$ for sbottom masses $m_{\tilde{b}} \gtrsim 70 \mathrm{GeV}$. This is because the relation (20) forces the stop squarks to be rather heavy $(\mathcal{O}(200) \mathrm{GeV})$. Only for gluinos 
of $\mathcal{O}(1) \mathrm{GeV}$ and $m_{\tilde{b}}=45 \mathrm{GeV}$ (the strict LEP limit on squarks) one can scarcely reach $\delta_{\tilde{g}} \simeq-3 \%$. As in Ref. [2], we have explicitly checked (Cf. Fig.4) that for our choices of the squark masses, the induced deviations of the $\rho$-parameter from 1 satisfy

$$
|\delta \rho| \leq 0.005
$$

The effect of the mixing is studied in Figs. 5-6. To illustrate the potentiality of this effect, it will suffice to concentrate on the stop mass matrix, where it is most likely to arise:

$$
\mathcal{M}_{\tilde{t}}^{2}=\left(\begin{array}{cc}
M_{\tilde{b}_{L}}^{2}+m_{t}^{2}+\cos 2 \beta\left(\frac{1}{2}-\frac{2}{3} s_{w}^{2}\right) M_{Z}^{2} & m_{t} M_{L R} \\
m_{t} M_{L R} & M_{\tilde{t}_{R}}^{2}+m_{t}^{2}+\frac{2}{3} \cos 2 \beta s_{w}^{2} M_{Z}^{2} \cdot
\end{array}\right) .
$$

For $M_{L R}=0$ one recovers eq.(19), but for nonvanishing $M_{L R}$ a light mass eigenvalue is possible. As a matter of fact, a light stop is still phenomenologically allowed [14], even below the purported lower LEP limit of $45 \mathrm{GeV}$ on all charged particles [15]. In general, once $\tan \beta$ and $m_{\tilde{b}}$ are fixed, we are free to choose two independent parameters in the stop mass matrix: $\left(M_{\tilde{t}_{R}}, M_{L R}\right)$, which, if desired, can be conveniently traded for $\left(m_{\tilde{t}_{1}}, \theta_{t}\right)$, $\left(m_{\tilde{t}_{1}}, M_{L R}\right)$ etc., $m_{\tilde{t}_{1}}$ being the lightest of the two mass eigenvalues. As for the mixing parameter $M_{L R}$ it is in principle arbitrary, with the caveat that

$$
M_{L R} \leq 3 m_{\tilde{b}_{L}}
$$

It roughly corresponds to a well-known necessary, though not sufficient, condition to avoid colour-breaking vacua [16]. In Fig.5 we display the dependence of $\delta_{\tilde{g}}$ on $M_{L R}$ for fixed $m_{\tilde{b}}=80 \mathrm{GeV}, \theta_{t}=\pi / 4$ and various gluino masses. We see that $\delta_{\tilde{g}}$ can be of either sign and reach the $\pm 2 \%$ level in the case of light gluinos. However, for large enough $M_{L R}$ (i.e. small enough $m_{\tilde{t_{1}}}$ ) we can approach regions where a few percent correction is also possible for $\mathcal{O}(100) \mathrm{GeV}$ gluinos. Finally, in Fig.6 we display the optimal situation where the strong SUSY corrections to the top quark width are the largest. Here we have fixed the rather conservative values $m_{\tilde{b}}=m_{\tilde{g}}=120 \mathrm{GeV}$ for the sbottom and gluino masses (compatible with the "traditional" CDF bounds [17]). In contrast to Fig.5, where $\theta_{t}$ was kept fixed at a specific value, we now let it vary by computing contour lines of constant $\delta_{\tilde{g}}$ in the $\left(M_{L R}, m_{\tilde{t}_{1}}\right)$-plane. In these conditions, there is a threshold (pseudo) singularity [18] (similar to the one found in Fig.5) associated to the wave-function renormalization of the top quark line at $m_{\tilde{t}_{1}}=54 \mathrm{GeV}$. We cannot arbitrarily approach from above the (dashed) threshold line in Fig.6 without breaking perturbation theory. Most remarkably, however, even staying prudentially away from it we can obtain $\mathcal{O}(-10) \%$ corrections. On the contrary, if we approach the threshold line from below (a non-singular limit), the correction is positive and of order $5 \%$. Nevertheless, it should be clear that the most 
interesting scenario for our decay corresponds to $\delta_{\tilde{g}}<0$, in which case the two-body supersymmetric decay $t \rightarrow \tilde{t_{1}} \tilde{g}$ is phase-space blocked up. For this situation the strong supersymmetric corrections could be reinforced by the additional negative contributions from the electroweak supersymmetric sector of the MSSM [2].

Some words on previous work are in order. In Ref. [19], a first study of $\delta_{\tilde{g}}$ as a function of $m_{t}$ and $m_{\tilde{g}}$ was presented. We qualitatively confirm their results. In that reference, however, the impact from the mixing and threshold effects were completely missed and only the simplest situation, characterized by degenerate masses, was considered 3 . Even in this case, i.e. adapting ourselves to the set of hypotheses and exact inputs considered in that reference, we found a relative deviation of $30-40 \%$ (our result being higher than theirs) f.

\section{Conclusions}

In summary, the strong supersymmetric corrections to the standard top quark decay mode $t \rightarrow W^{+} b$ are potentially large: to wit, of order $+5 \%$ to $-(5-10) \%$. Remarkably enough, the most significant signature appears for sufficiently heavy gluinos (compatible with conservative CDF limits) and in the presence of an intermediately heavy stop squark $\tilde{t}_{1}$ with a mass slightly beyond the threshold for the direct supersymmetric decay $t \rightarrow \tilde{t_{1}} \tilde{g}$, i.e. for $m_{\tilde{t}_{1}}=\mathcal{O}(50-60) G e V$. However, even for $m_{\tilde{t}_{1}}=\mathcal{O}(80-100) G e V$ the correction could remain of order $-5 \%$. The case $\delta_{\tilde{g}}<0$ is specially significant in that it would add up to the conventional QCD corrections $\left(\delta_{Q C D} \simeq-8 \%\right)$ and in favourable circumstances the total strong correction could reach $-(15-20) \%$. Last but not least, as this correction is insensitive to $\tan \beta$, we may envisage a situation with large $\tan \beta \geq m_{t} / m_{b}$ in which the electroweak supersymmetric corrections [2], being also negative, are of the same order of magnitude as the ones studied here, in which case the total MSSM correction to the top quark width (the Higgs correction being negligible [8]) could result in a remarkable reduction of $\Gamma\left(t \rightarrow W^{+} b\right)$ by about $25 \%$.

\section{Acknowledgements:}

The work of RJ and JS has been partially supported by CICYT under project No. AEN93-0474.

\footnotetext{
${ }^{3}$ Notice that the assumption of stop masses equal to sbottom masses is incompatible with eq.(20).

${ }^{4}$ We carefully checked this discrepancy by performing two completely independent computations of $\delta_{\tilde{g}}$ and got perfect agreement with the alternative results presented here.
} 


\section{References}

[1] H. Nilles, Phys. Rep. 110 (1984) 1; H. Haber and G. Kane, Phys. Rep. 117 (1985) 75; A. Lahanas and D. Nanopoulos, Phys. Rep. 145 (1987) 1; See also the exhaustive reprint collection Supersymmetry (2 vols.), ed. S. Ferrara (North Holland/World Scientific, Singapore, 1987); H.E. Haber, Introductory Low-Energy Supersymmetry, preprint SCIPP-92/33, Proc. of the 1992 TASI, Univ. of Colorado.

[2] D. Garcia, W. Hollik, R.A. Jiménez and J.Solà, Nucl. Phys. B427 (1994) 53.

[3] J.M. Yang and C.S. Li, Phys. Lett. B320 (1994) 117.

[4] M. Jeżabek and J.H. Kühn, Nucl. Phys. B314 (1989) 1; ibid B320 (1989) 20; C.S. Li, R.J. Oakes and T.C. Yuan, Phys. Rev. D43 (1991) 3759.

[5] A. Denner and T. Sack, Z. Phys. C46 (1990) 653; Nucl. Phys. B358 (1991) 46.

[6] G. Eilam, R.R. Mendel, R. Migneron and A. Soni, Phys. Rev. Lett. 66 (1991) 3105.

[7] B.A. Irwin, B. Magnolis and H.D. Trottier, Phys. Lett. B256 (1991) 533; T.C. Yuan and C.-P. Yuan, Phys. Rev. D44 (1991) 3603.

[8] B. Grządkowski and W. Hollik, Nucl. Phys. B384 (1992) 101.

[9] A. Denner and A.H. Hoang, Nucl. Phys. B397 (1993) 483.

[10] M. Böhm, H. Spiesberger and W. Hollik, Fortschr. Phys. 34 (1986) 687; W. Hollik, Fortschr. Phys. 38 (1990) 165; W. Hollik, in: Precision Tests of the Standard Model, ed. P. Langacker (Advanced Series on Directions in High Energy Physics, World Scientific, 1994).

[11] A. Denner, Fortschr. Phys. 41 (1993) 307.

[12] F. Abe et al. (CDF Collab.), Phys. Rev. Lett. 73 (1994) 225.

[13] L. Clavelli, Phys. Rev. D46 (1992) 2112; L. Clavelli, P. Coulter and K. Yuan, Phys. Rev. D47 (1993) 1973; M. Jeżabek and J.H. Kühn, Phys. Lett. B301 (1993) 121; J. Ellis, D.V. Nanopoulos and D.A. Ross, Phys. Lett. B305 (1993) 375.

[14] P. Abreu, et al. (DELPHI Collab.), Phys. Lett. B247 (1990) 148; O. Adriani, et. al. (L3 Collab.), Phys. Rep. 236 (1993) 1.

[15] T. Kon and T. Nonaka, Phys. Lett. B319 (1993) 355. 
[16] J.M. Frère, D.R.T. Jones and S. Raby, Nucl. Phys. B222 (1983) 11; J. Ellis, D.V. Nanopoulos and K. Tamvakis, Phys. Lett. B121 (1983) 123; M. Claudson, L. Hall and I. Hinchliffe, Nucl. Phys. B228 (1983) 501; J.F. Gunion, H.E. Haber and M. Sher, Nucl. Phys. B306 (1988) 1.

[17] F. Abe et al., (CDF Collab.) Phys. Rev. Lett. 69 (1992) 3439.

[18] T. Bahattacharya and S. Willenbrock, Phys. Rev. D47 (1993) 4022.

[19] C.S. Li, J.M. Yang and B.Q. Hu, Phys. Rev. D48 (1993) 5425. 


\section{Figure Captions}

- Fig.1 Feynman diagrams, up to one-loop order, for the SUSY-QCD corrections to the top quark decay $t \rightarrow W^{+} b$. Each one-loop diagram is summed over the mass-eigenstates of the stop and sbottom squarks $\left(\tilde{b}_{a}, \tilde{t}_{b} ; a, b=1,2\right)$.

- Fig.2 Dependence of $\delta_{\tilde{g}}$ on (a) $m_{\tilde{g}}$ for $m_{t}=174 \mathrm{GeV}$ and a wide range of SUSY masses including the light gluino region, and on (b) $m_{t}$ (within the CDF limits) for the same squark masses as in (a) and $m_{\tilde{g}}=m_{\tilde{b}}$. In both cases we assume no squark mixing and $\tan \beta=1$.

- Fig.3 $\delta_{\tilde{g}}$ as a function of $\tan \beta$, for the same squark and gluino masses as in Fig.2a, and $m_{t}=174 \mathrm{GeV}$.

- Fig.4 Deviation of the $\rho$-parameter from 1 for the squark masses used to evaluate $\delta_{\tilde{g}}$ in Fig.2(a).

- Fig.5 Plot of $\delta_{\tilde{g}}$ versus the mixing parameter of the stop mass matrix, $M_{L R}$, for $\theta_{t}=\pi / 4, m_{\tilde{b}}=80 \mathrm{GeV}$ and various gluino masses $\left(m_{t}=174 \mathrm{GeV}\right)$.

- Fig.6 Contour plots of $\delta_{\tilde{g}}$ in the $\left(M_{L R}, m_{\tilde{t_{1}}}\right)$-plane for $m_{\tilde{b}}=m_{\tilde{g}}=120 \mathrm{GeV}$. The mixing angle $\theta_{t}$ varies accordingly and the shaded area is excluded by the condition $M_{\tilde{t}_{R}}^{2}>0$ in the stop mass matrix $\left(m_{t}=174 \mathrm{GeV}\right)$. 
This figure "fig1-1.png" is available in "png" format from: http://arxiv.org/ps/hep-ph/9503398v1 

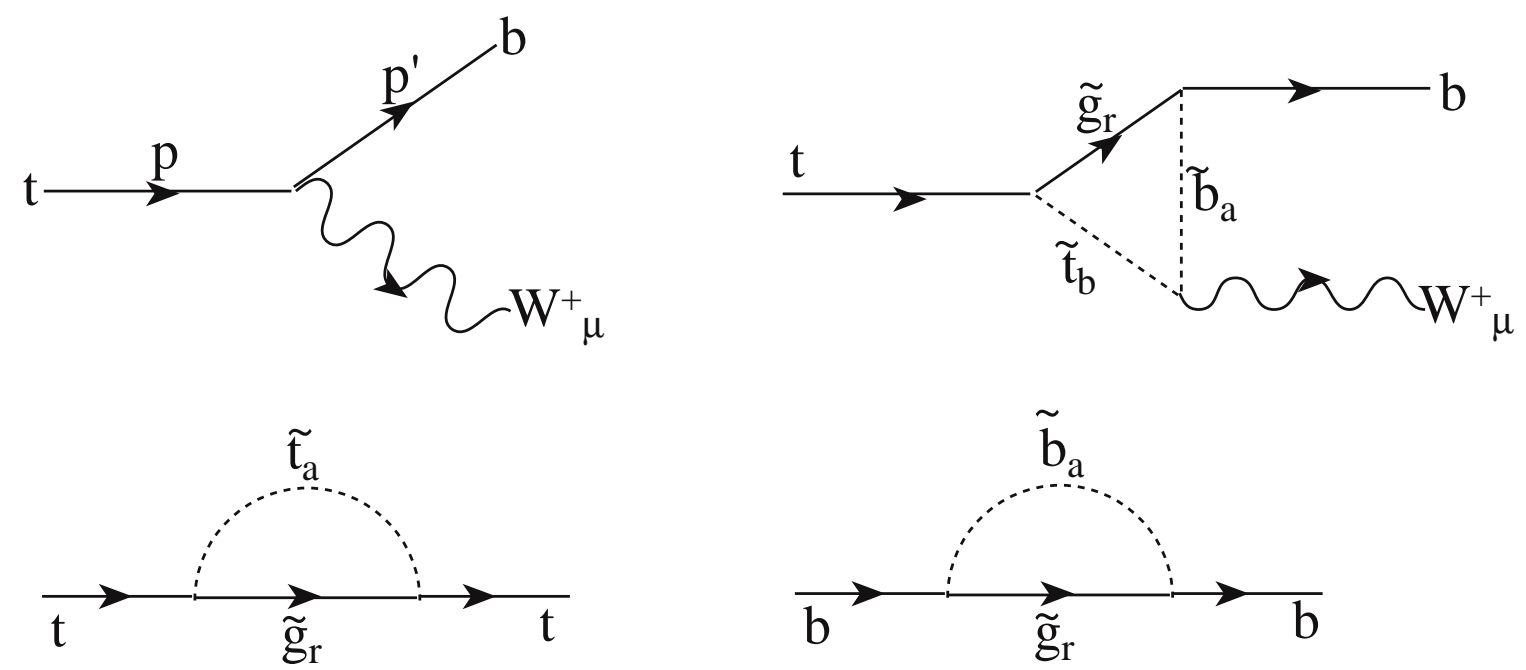

Fig. 1 
This figure "fig2-2.png" is available in "png" format from: http://arxiv.org/ps/hep-ph/9503398v1 


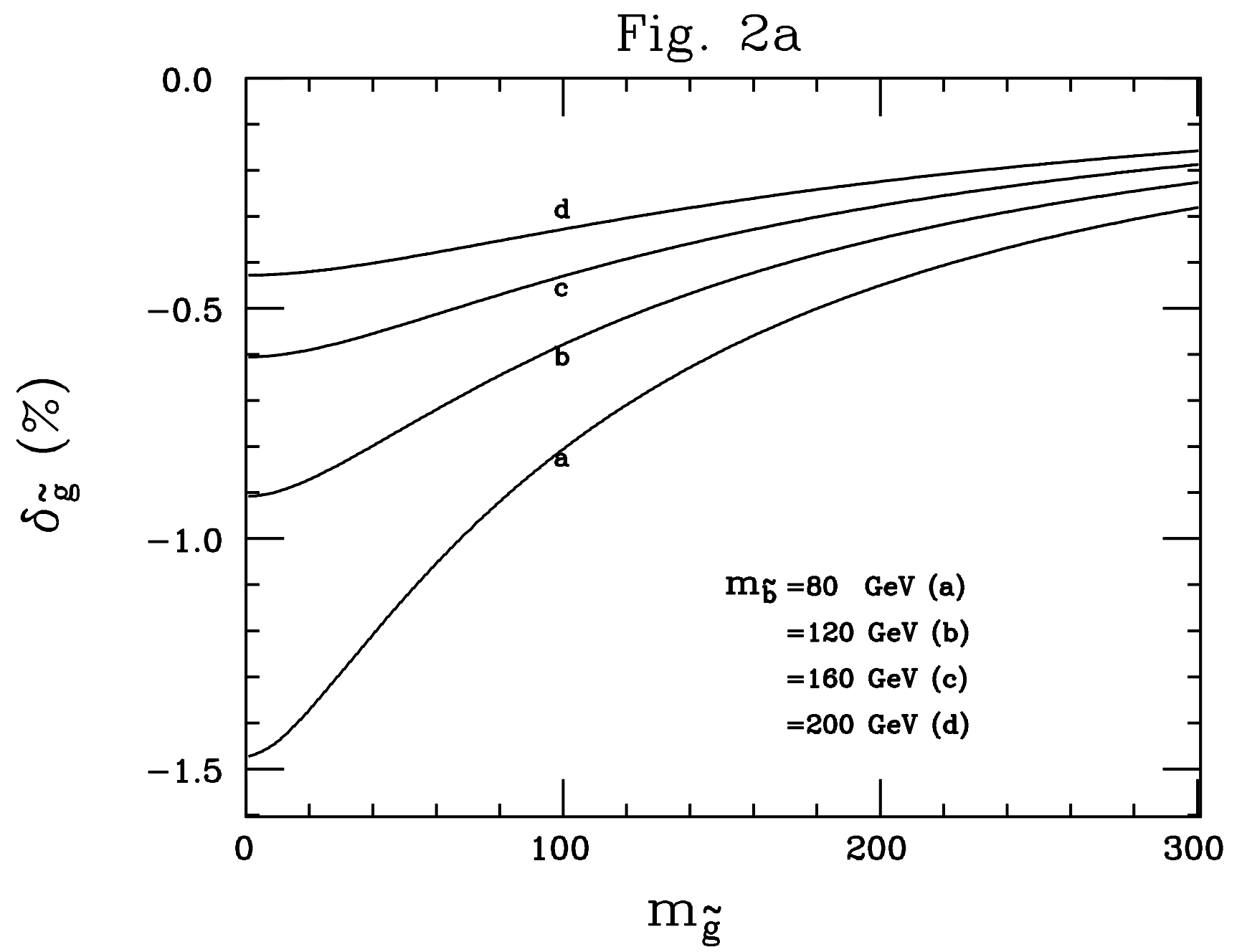


Fig. 2b

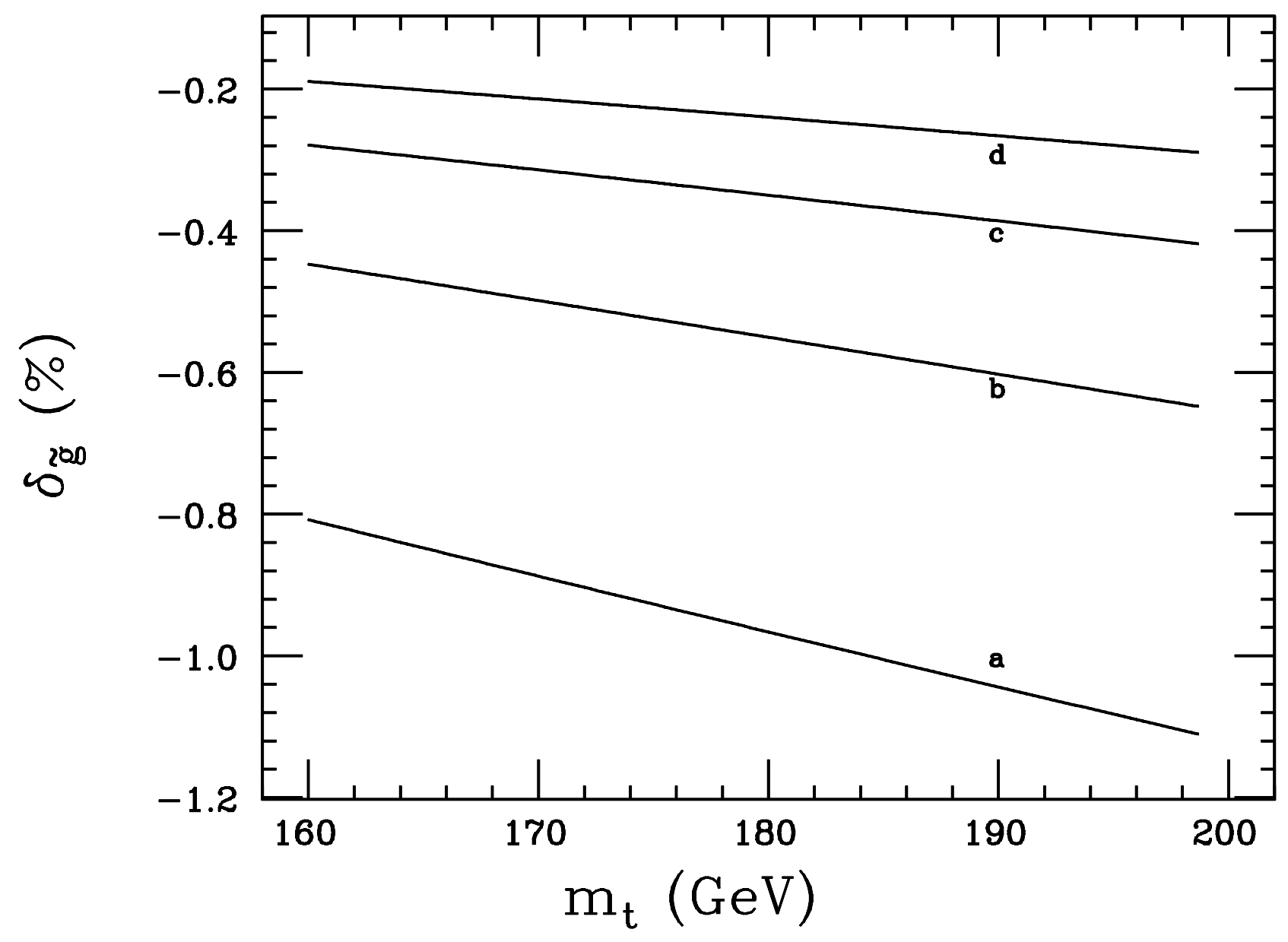


This figure "fig2-3.png" is available in "png" format from: http://arxiv.org/ps/hep-ph/9503398v1 


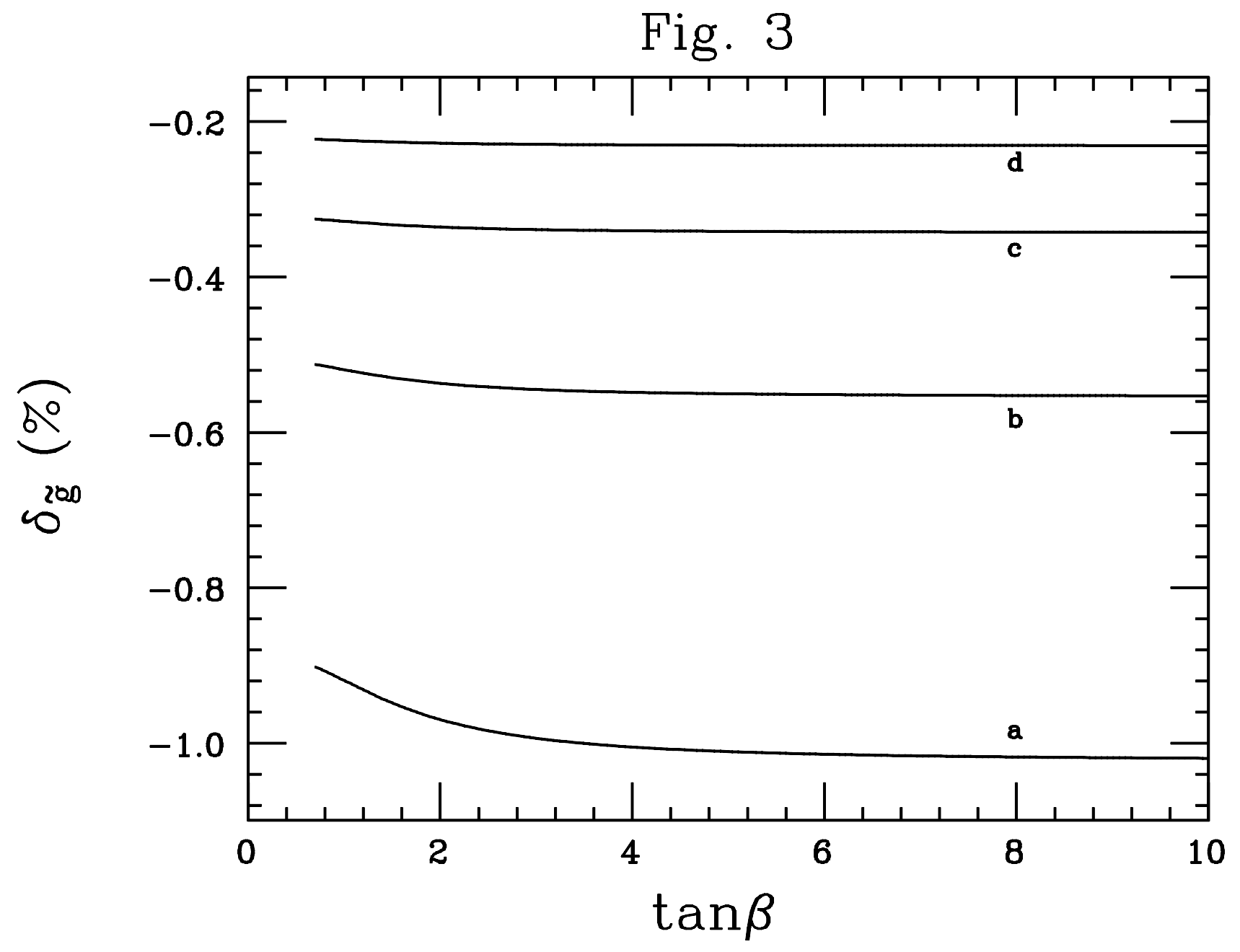


This figure "fig2-4.png" is available in "png" format from: http://arxiv.org/ps/hep-ph/9503398v1 


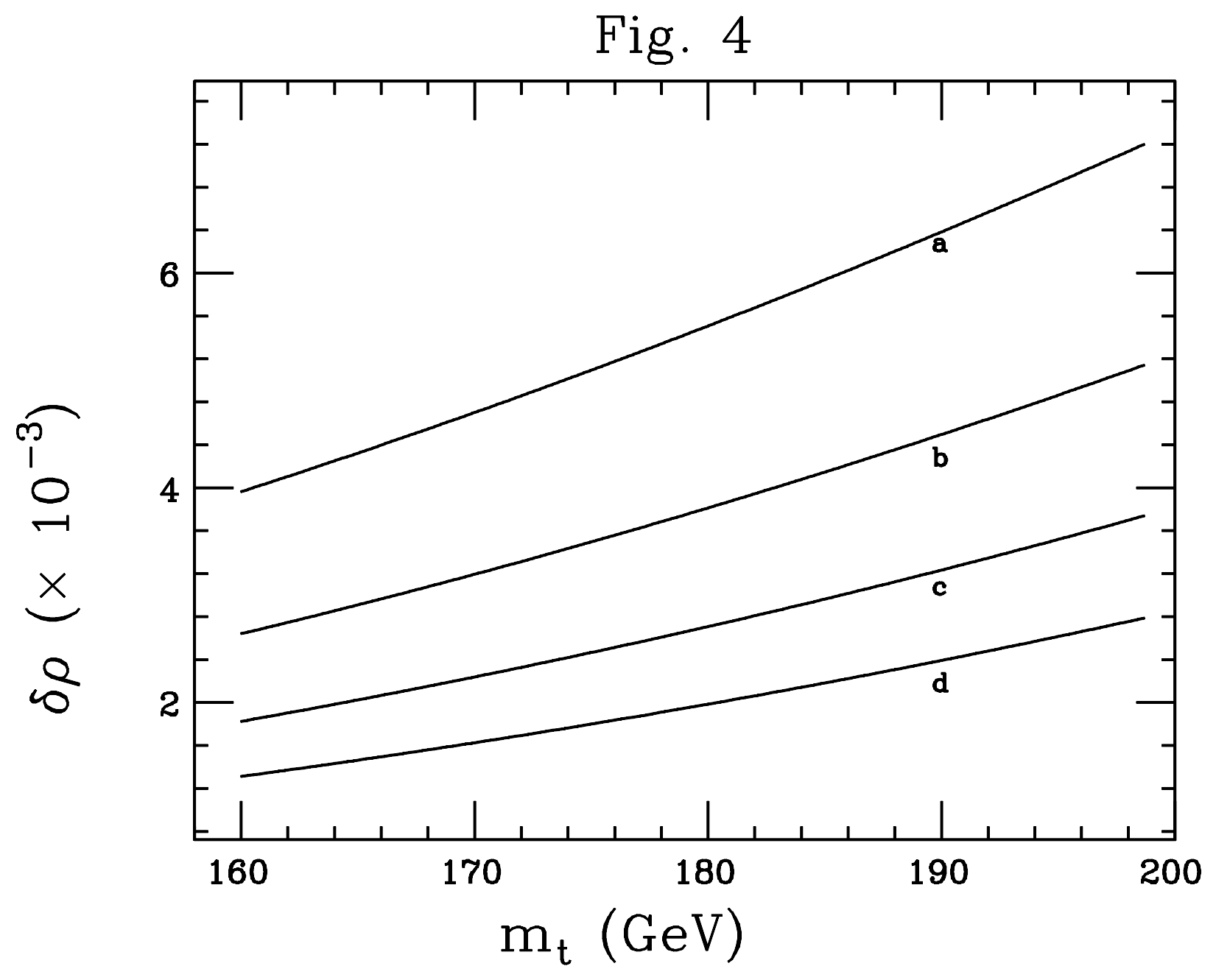


This figure "fig2-5.png" is available in "png" format from: http://arxiv.org/ps/hep-ph/9503398v1 


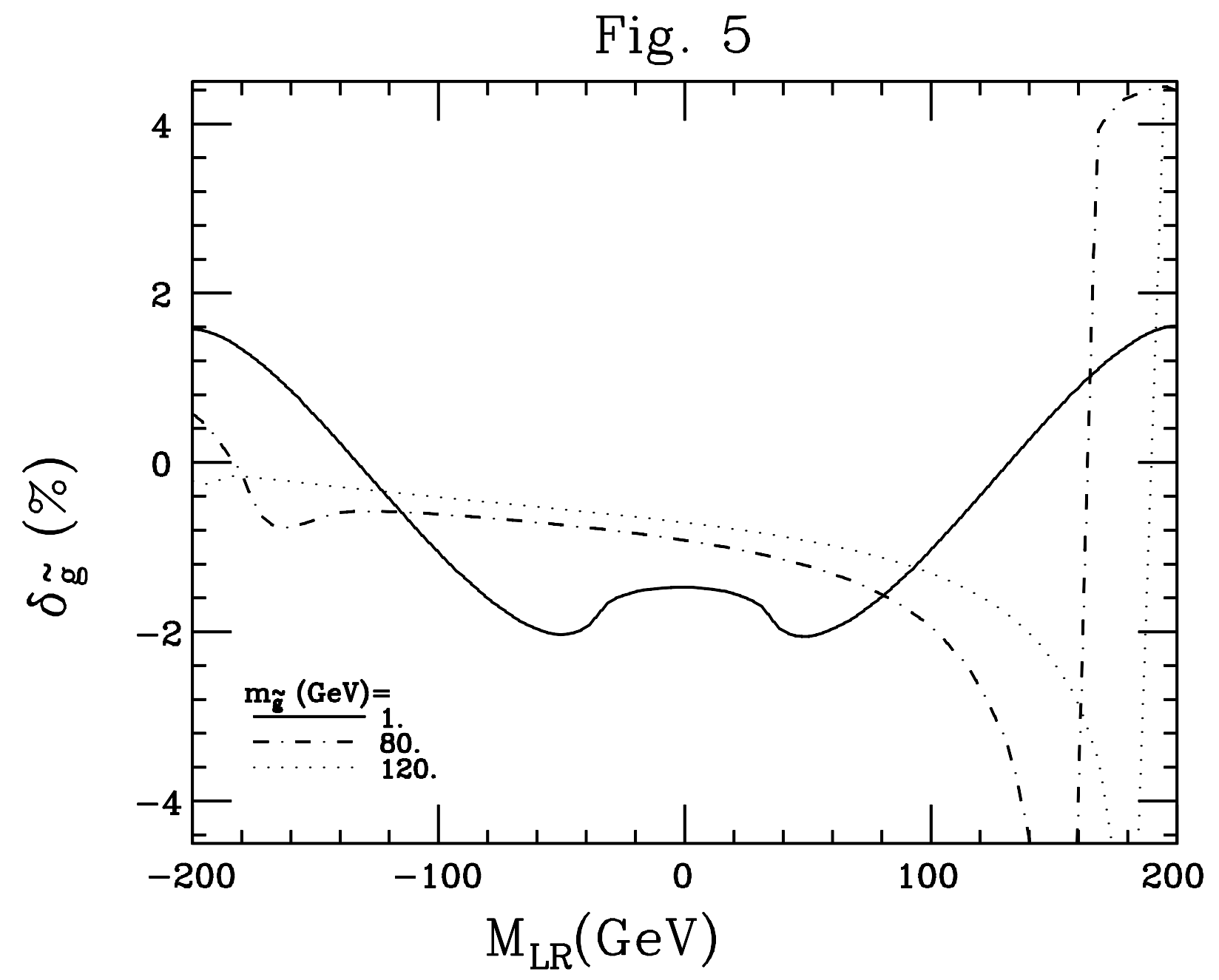


This figure "fig2-6.png" is available in "png" format from: http://arxiv.org/ps/hep-ph/9503398v1 


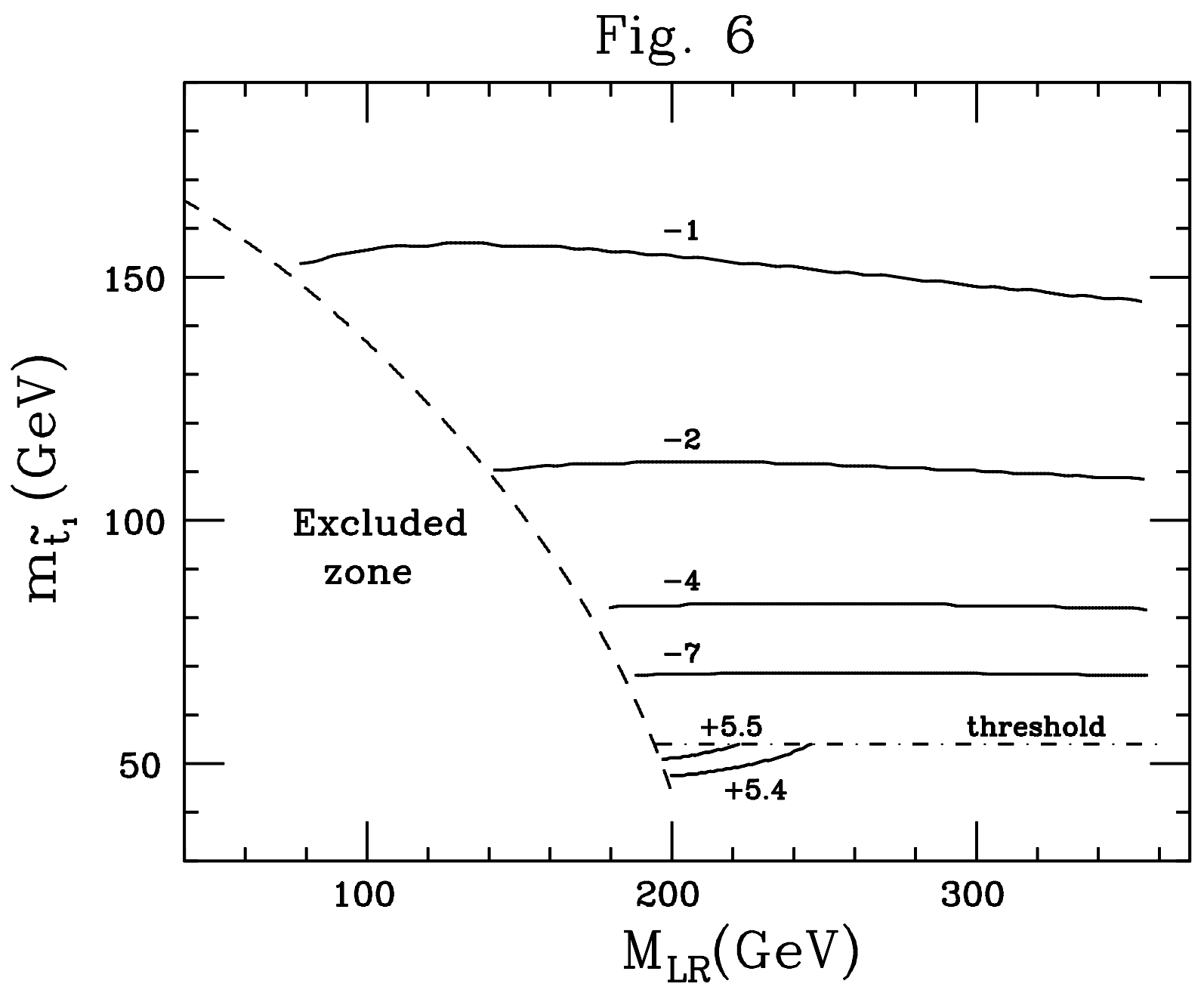


This figure "fig2-7.png" is available in "png" format from: http://arxiv.org/ps/hep-ph/9503398v1 